\title{
Proposal for capturing patient experience through extended value frameworks of health technologies
}

\author{
András Inotai, PhD; Ivett Jakab, MSc; Diana Brixner, PhD; Jonathan D Campbell, PhD; Neil Hawkins, PhD; \\ Lars Erik Kristensen, MD, PhD; Mata Charokopou, MSc; Irina Mountian, MD, PhD; \\ Boglarka Szegvari, MSc; and Zoltán Kaló, PhD
}

\section{What is already known about this subject}

- Patient experience is increasingly considered as an important aspect of the value of a health technology.

- However, there is no consistent or standardized approach to help manufacturers and healthcare decision makers ensure health technology assessments incorporate patient experience fairly.

\author{
What this study adds \\ - This study used the results of a \\ systematic literature review and expert \\ focus groups to define five potential \\ patient experience domains that \\ could be used to assess the patient \\ experience-related value of a health \\ technology. \\ - The framework was developed to be \\ flexibly implemented to allow it to be \\ used as an extension of existing value \\ frameworks or to create new patient \\ experience-focused value frameworks.
}

\author{
Author affiliations \\ András Inotai, PhD; Ivett Jakab, MSc; and \\ Zoltán Kaló PhD; Syreon Research Institute, \\ Budapest, Hungary. András Inotai, PhD; \\ and Zoltán Kaló PhD; Center of Health \\ Technology Assessment, Semmelweis \\ University, Budapest, Hungary. Diana Brixner, \\ PhD; University of Utah, Salt Lake City, UT. \\ Jonathan D Campbell, PhD, University of \\ Colorado, Aurora, CO. Neil Hawkins, PhD, \\ University of Glasgow, Glasgow, United \\ Kingdom. Lars Erik Kristensen, MD PhD, The \\ Parker Institute, Copenhagen University \\ Hospital, Bispebjerg and Frederiksberg, \\ Copenhagen F, Denmark. Mata Charokopou, \\ MSc; Irina Mountian, MD PhD; and Boglarka \\ Szegvari, MSc; UCB Pharma, Brussels, \\ Belgium.
}

\section{AUTHOR CORRESPONDENCE: \\ Diana Brixner, 801.581.3182; \\ diana.brixner@utah.edu}

J Manag Care Spec Pharm 2021;27(7):936-47

Copyright $\odot 2021$, Academy of Managed Care Pharmacy. All rights reserved.

including HTA experts and patient representatives used an iterative process to develop potential value domains to capture PEx, in accordance with international guidelines. Subsequently, a panel of international payer experts was used to challenge the proposed PEx domains and provide recommendations for implementation. 
RESULTS: The SLR found 61 VFs and multi-criteria decision analyses (MCDAs) that considered PEx; however, PEx-related value elements were often referred to superficially, without clear definitions. Five potential PEx domains, with proposed measures for each, were developed and refined using expert feedback: (1) responsiveness to patient's individual needs, (2) improved health literacy and empowerment, (3) patient and caregiver reported outcomes, (4) household's financial burden, and (5) improved access for vulnerable patient populations. A flexible approach for framework implementation was proposed.

CONCLUSIONS: Proposed PEx domains could be implemented at multiple levels of healthcare decision making to formalize consideration of PEx in the assessment of value, either through the extension of existing VFs or to create new PEx-focused VFs and more holistic decision making tools.

Health technology assessment (HTA) is a multidisciplinary field of policy analysis, which considers the long- and shortterm medical, social, ethical, and economic implications of the development, diffusion, and use of health technologies (eg, pharmaceuticals, medical devices, combined technologies). ${ }^{1,2}$ The purpose of HTA is to provide policymakers with available information about new and established health technologies to inform resource allocation decisions across different settings.

The majority of HTA systems consider three traditional value pillars in judging the value of new health technologies. First, differential clinical value dominantly measured by healthcare professionals in clinical trials is considered. This is complemented by health-related quality of life (QOL) improvement reported by patients through standardized and validated instruments. ${ }^{3}$ Finally, to reflect budget constraints, estimated changes in direct healthcare costs are also considered. In several HTA systems, these traditional pillars are aggregated in the incremental cost per incremental quality-adjusted life-years (QALY) ratio, which is compared to a financial threshold to judge the economic value of health technologies. ${ }^{4}$

However, recently, the narrow scope of traditional HTA value domains has become obvious in many jurisdictions, as patient centricity has become a common objective in most developed health systems globally. Patient centricity can be facilitated by two components: firstly, the timely involvement of patient representatives to healthcare research, product development, care delivery and health policies. Secondly, by the establishment of transparent and verifiable patient experience (PEx)-related criteria in the value judgment of marketed health technologies. Of note, PEx is also considered to be increasingly important from regulatory perspective, see the FDA guidance on Patient Experience Data. ${ }^{5}$ This current paper, however, focuses on capturing PEx for marketed technologies with broad health, economic and social perspective.

From the abovementioned three traditional pillars of value judgment by HTA systems, health-related QOL addresses some aspects of PEx. However, many aspects of what matters to patients, such as reduced burden on patients' households or factors contributing to improved patient adherence and persistence, cannot be easily captured by focusing only on clinical trial data and changes in direct healthcare costs and QALYs. Similarly, societal value propositions of new technologies, such as improved equity or labour productivity, necessitate extension of the scope of value judgment by HTA systems. As a consequence, there is an ongoing global movement towards extending the scope of HTA, mainly by including new value domains. ${ }^{6-9}$ Extended value frameworks (eVFs) aim to list all aspects of value outside of those commonly captured by traditional cost-effectiveness metrics considered during the decisionmaking process to inform HTA. ${ }^{10}$ The extension of value judgement with new elements creates an opportunity for more patient-centric evaluation of health technologies.

Patient perspective can be incorporated at different levels of decision making in healthcare. ${ }^{11}$ At the micro level, where decisions are about individual patients' treatment, shared decision making may help patients consider different options and achieve informed preferences (eg, as intended by the American Society of Clinical Oncology [ASCO] Value Framework). ${ }^{12-14}$ At the mezzo level, patients can be involved in hospital-level decision making (eg, formulary listing) or in the development of clinical guidelines. At the macro level, the patient perspective can be considered within research and development, regulatory, pricing and reimbursement decisions. However, involvement of patients into clinical decisions and patient representatives to policy recommendations is still ad hoc in many jurisdictions (eg, countries and regions, decision bodies, different types of health technologies, disease areas).

Alongside these efforts, many organizations are currently moving patient centricity forward in different fields of healthcare; however, this progress is heterogeneous and without any centralised aims. ${ }^{15-26}$ Some of these organizations have started to develop VFs by considering various additional outcomes that explore direct (eg, patient and caregiver health related QOL, patient satisfaction, economic impact) or indirect (non-adherence of patients) feedback from patients and their households. ${ }^{27}$ On the other hand, consideration of patient perspective varies across different jurisdictions. Moreover, the methodology used 
to generate evidence beyond standard clinical and direct medical cost data is associated with challenges, making it difficult to consider these in decision making in a transparent and reproducible way. ${ }^{28-30}$ For value assessment, international guidelines recommend transparent and/or scientifically rigorous selection, definition, measurement, and weighting of value attributes. ${ }^{31}$ However, patient relevant value attributes in VFs often contain no description of selection methodology, consensus definition on attributes or guidance on measurement. Currently, along with the development of new VFs, there is momentum to improve patient centricity by including PEx in decision making, and to promote and amplify discussion on PEx elements and measurement. ${ }^{32,33}$

Overall, the global process of extending existing VFs with PEx is heterogenous and not harmonized across countries and decision-makers. Hence, there is a risk that an even more divergent global system is created, which then must be adhered to by technology developers. Because of the differences in expectations by HTA and payer bodies, pharmaceutical companies may only focus on the needs of their most important markets in the evidence generation process. Additionally, the expectations of all HTA and payer bodies are changing rapidly. There is a need for harmonization in the extension of VFs, with special focus on PEx-related value domains.

The aim of this study was to develop a proposal for reducing the heterogeneity of including PEx in extended HTA frameworks. The study objectives were to: (1) review how published frameworks capture PEx-related value propositions for health technologies, including pharmaceuticals, medical devices, and combined technologies; (2) create a "PEx framework" consisting of mutually exclusive PEx domains with definitions and measurement options; and (3) identify whether there are differences between potential PEx domains used within HTA frameworks at different (micro/mezzo/macro) levels of decision making.

\section{Methods}

\section{SYSTEMATIC LITERATURE REVIEW (SLR) ON EXISTING VFS AND MULTICRITERIA DECISION ANALYSES TO EVALUATE HOW PEX IS CAPTURED}

As there is no consensus on a definition for PEx, ${ }^{34}$ it was likely that articles implicitly incorporating PEx would have been missed using pre-defined PEx search terms. To overcome this challenge, a reverse approach was used for the SLR, searching for all value elements listed by VFs and then selecting those possibly relevant to PEx. This method also ensured articles found, and any resulting frameworks suggested, were relevant to current decision-making algorithms, with the intention of reducing the heterogeneity of newly developed frameworks.

Searches were performed on Scopus, Medline (via PubMed), PROSPERO and Cochrane databases on the 6th October 2018 and were completed according to the PRISMA guidelines. ${ }^{35}$ (Supplementary Table 1, available in online article). Defining value elements in healthcare is a dynamic field; this SLR aimed to capture more recent approaches. There has been a steep increase in the number of multicriteria decision analysis (MCDA) publications since 2014, ${ }^{36}$ and so the search timeframe was restricted from 2013 onwards. No geographical restrictions were applied; however, only English language records were included. Title and abstract screening, followed by full-text screening, of the shortlisted articles was performed independently by two reviewers; conflicts were resolved by a third independent reviewer. All data extracted were double-checked by a second reviewer. An additional search of the references of eligible full-text papers and other SLRs identified was completed to capture any missed records. In addition, a targeted review on nonexhaustive list of the websites of organizations involved in developing patient-centric VFs was also conducted (Supplementary Table 2, available in online article).

For data extraction, the original wording from the articles describing VF value elements was extracted to formulate PEx domains; stratifications were made according to different decision levels (micro, mezzo, and macro level), and relevance to pharmaceuticals vs non-pharmaceuticals (medical devices, healthcare programs, etc.) by the abstract reviewers.

\section{DEVELOPMENT OF PEX DOMAINS}

Domains aiming to capture PEx (with definitions and proposed measurements) were developed iteratively by the multidisciplinary core research team through a multi-round deliberative process moderated by the research leader to facilitate consensus. The core research team included two patient representatives who had previous training in HTA terminology and were active in non-disease specific patient advocacy at an international level, two HTA experts (one from Europe and one from the United States) and two experts from academia with prior experience of developing and adapting VF/MCDAs in different settings. Including patient representatives in the core research team from the beginning of framework development ensured patient opinion was incorporated at all stages of development, in line with previously published recommendations. ${ }^{37}$

Iterative development included the following steps: (1) all potential PEx-related value propositions were extracted from the articles by abstract reviewers; (2) similar 


\section{FIGURE 1 Flow of Information Diagram}

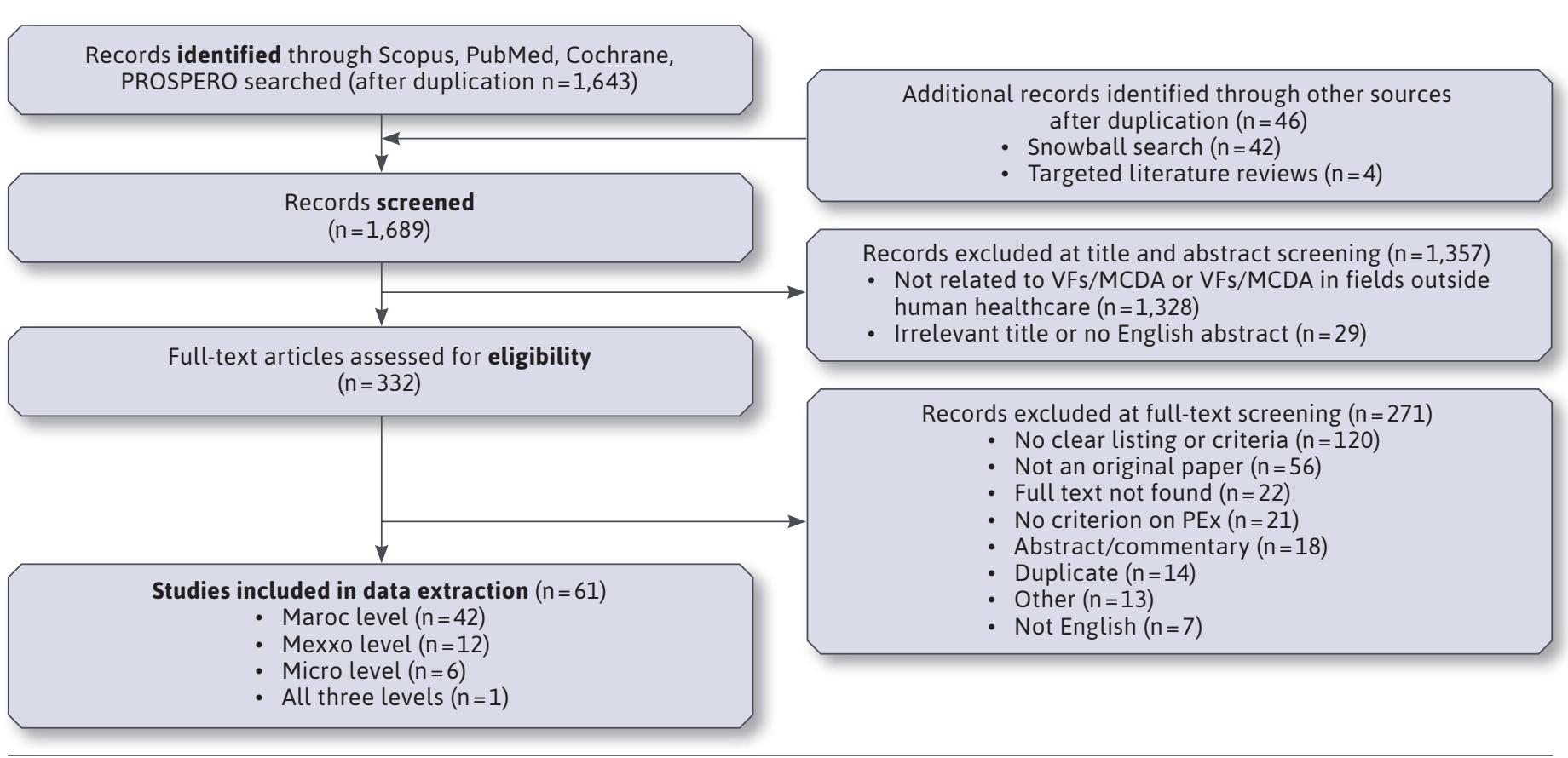

$M C D A=$ multiple criteria decision analysis; $P E x=$ patient experience; $V F s=$ value frameworks.

topics were clustered into expression clouds by two senior researchers independently, with conflicts resolved on mutual agreement; (3) umbrella terms were created for each expression cloud and proposed as potential PEx domain names by the core research team; (4) potential PEx domains were evaluated for overlaps, feasibility of measurement and implementation, and data availability by the core research team; and (5) the number of PEx domains was reduced to ensure non-redundancy, preference independence and to minimize overlaps according to international recommendations by the core research team. ${ }^{38-40}$

Once PEx domains had been drafted, a panel was set up with additional international payer experts (two from the United States, one each from Germany, Scotland, and Denmark). This panel was set up to provide an opportunity for experts to challenge the proposed approach from the perspective of decision makers (as potential future users) and suggest recommendations on the dissemination and implementation of the proposed PEx domains. The faceto-face panel discussion aimed to achieve consensus. Pre-reading was presented to the external advisors in a preparatory teleconference prior to the live discussion. Finally, based on the expert panel feedback, the initial set of PEx domains were revised by the core research team and then reviewed again by the experts at a follow-up teleconference. The development and resulting set of PEx domains are described below.

\section{Results}

The SLR and additional targeted review identified 61 VFs/ MCDAs with a published list of domains including potential PEx-related criteria (Figure 1); 42 were macro-level decision-making, 12 were mezzo-level decision-making, and 6 were micro-level decision-making. One patient perspective VF for the evaluation of the patient centricity of other VFs was identified which could be applied to all three levels (Supplementary Table 3, available in online article). ${ }^{21}$ No published SLRs on PEx domains in VFs/MCDAs were identified, however, one SLR article reviewed the involvement of patients during the development of VFs. ${ }^{28}$

Within the identified domains referring to PEx in VFs and MCDAs (per original wording of the articles), PEx and patient centricity was often associated with very general or superficial domains (eg, "quality of life" or "equity aspect"). ${ }^{41-45}$ Similar domains or concepts were 


\section{FIGURE 2 Merging and Selection of PEx Domains}

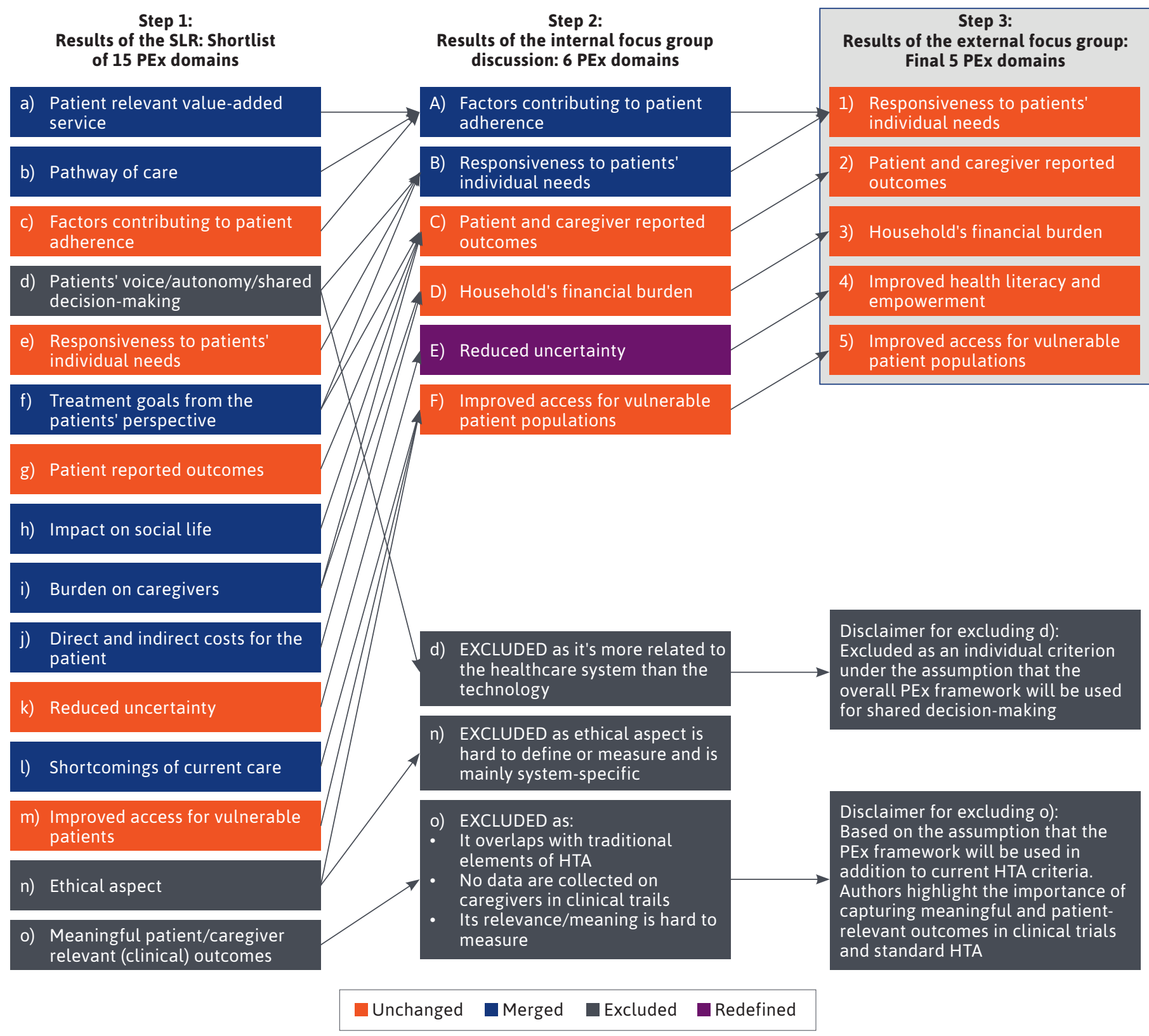

$H T A=$ health technology assessment; $P E x=$ patient experience; $S L R=$ systematic literature review.

often described with multiple terms (eg, patient-reported outcomes, quality of life, functioning, disease symptoms, disease-related distress, disease impact on social and daily life). Often no formal or detailed definition was provided for PEx domains (eg, articles referred to "ethical aspect"). ${ }^{43-45}$
Some PEx domains captured both causes and effects which could result in double counting (eg, ease of use [as a cause] vs improved patient adherence [as an effect]). Measurements of potential PEx domains in the identified studies were often not reported or reported inconsistently 
and without detail. ${ }^{46}$ In many MCDAs, a categorical scale (eg, no, minor, moderate, major improvement) was used. ${ }^{47}$

\section{ITERATIVE DEVELOPMENT OF PEX DOMAINS}

A shortlist of 15 PEx domains (Figure 2, column 1) was developed and discussed by the core research group. It was assumed that the proposed PEx domains would most often be considered as add-ons to the traditional elements of value, unless the VF had already been extended with similar domains. The domains would be flexible and adaptable to the changing field of patient perspective in HTA decision making. However, there may be potential overlap with existing elements (eg, patient-reported outcomes could fall into PEx domains and existing value domains, such as health gain). To ensure simplicity and feasibility, the number of domains were then reduced. The detailed description of the merging and exclusion process to generate an initial list of six PEx domains (Figure 2, column 2) was presented to, and challenged by, a panel of international payer experts. International experts recommended one additional merging of two PEx domains and edits to the names of some domains during the face-to-face panel discussion and the follow-up teleconference, which resulted in a list of five final potential PEx domains (Figure 2, column 3). The justifications (including what each domain adds to traditional value measurements, such as QALYs), definitions and incentives for use of the final five proposed PEx domains are presented in Table 1. The proposed measurements to be used for each domain are presented in Table 2 (PEx domains are not ranked in order of importance).

\section{Discussion}

Current value judgement methods in different jurisdictions are limitedly patient-centered. Recent efforts have been made to better capture what is relevant for patients to ensure healthcare research, product development, care delivery and health policies are more patient-centric. ${ }^{48}$ This is important as patients are the end-user beneficiaries of health care and their complex judgment of value may differ from clinicians' and decision-makers' judgment, both in terms of elements of value and their relative importance..$^{49}$ To increase patient centricity in healthcare systems, payers and policy-makers should understand the potential tradeoffs that patients may be willing to make in their treatment performance for an improved overall experience. ${ }^{50}$ The proposed framework takes into consideration current PEx related elements in decision-making processes and could be used as an add-on to traditional value elements (eg, those expressed through QALYs) in available HTA frameworks. Therefore, complementary patient centric value domains could expand the perspective of value judgement. Although some overlap and dependence/interrelation between criteria is unavoidable, we aimed to develop a framework that was as complete as possible by capturing all relevant factors, while minimizing the interdependence, redundancy and overlap of domains. . $^{38,39}$

There are multiple benefits associated with developing a structured framework of PEx domains. For example, it may help guide manufacturers to develop more patient-centered health technologies by increasing the predictability of their return on investment through transparent criteria and allowing manufacturers to plan data collection. Additionally, the framework incentivizes manufacturers to measure PEx and generate related evidence for the HTA process. Patients may contribute to both decision making about their own treatment and current HTA decision-making processes if asked for their systematic input based on these PEx domains. Finally, it may facilitate earlier patient engagement in HTA, as the measurement of PEx must be started before the HTA process, as early as during pipeline development.

Country- or even population-specific adaptation of PEx domains is needed. It is not expected that every country would consider all proposed PEx domains for all therapeutic areas or patient populations in the same way. Prior to implementation, each HTA system should assess the extent to which these PEx domains are already included, and the weight they are currently given, in their decision-making processes. There are multiple options for including (additional) PEx domains in the decision-making process. For example, the domains could be used to support deliberative decision-making processes by standardization of PEx aspects, or as a list of value attributes to consider if aiming to understand the impact of health technologies on patients in a holistic way. PEx can be integrated within existing HTA frameworks through augmented cost-effectiveness analyses, by including the PEx domains in the calculation of incremental costs or effectiveness, or using them as threshold modifiers. ${ }^{10}$ Finally, the domains could be incorporated into existing or future MCDA tools with an explicit decision rule to guide decision making. ${ }^{40}$ If multiple mutually exclusive PEx domains are applied at any decision level (macro, mezzo, micro), a standard definition and measurement is necessary for each domain. Although Table 2 proposes an indicative measurement for the PEx value domains, final instructions for PEx measurement in different domains should be concluded in each jurisdiction only after using them in the real world for a pilot period.

Because of the small sample size of VFs targeting nonpharmaceutical technologies and mezzo and micro level VFs, subgroup analyses according to health technology and 


\section{TABLE 1 Justification, Definitions and Incentives For Use of the Five Proposed PEx Domains}

PEx domain Justification, definition and incentives for use

Justification for domain: The needs and expectations of patients are heterogeneous, and patients may value different aspects of a health technology (e.g. survival likelihood, treatment burden, quality of life). ${ }^{32} \mathrm{~A}$ value-added service can have the potential to improve adherence to therapy in some patients but may not be relevant for others. The assumption that patient values are uniform and unchanging across patient populations is no longer supported. ${ }^{51}$ This domain aims to incentivize industry to consider how a new technology could answer the individual needs of patients.

Responsiveness

to patients'

Definition: Patients are different, for example, in pharmacokinetic profiles, demographics, comorbidities, socio-economic individual needs factors and individual preferences to accept severe side-effects or take higher risks for a curative therapy. An adequate response to individual (clinical and non-clinical) needs may be achievable, for example, through different dosing, absorption, drug interactions, administration form, side-effect profile, treatment plan, etc.

Incentive for manufacturers: 1) To develop health technologies which consider the heterogeneity of patients, and 2) to evaluate the magnitude of these benefits for different subgroups of patients.

Justification for domain: Health literacy is the degree to which individuals have the capacity and motivation to obtain, process and understand basic health information to make health decisions. ${ }^{22}$ Health literacy skills can empower patients to be involved in shared decision making and to allow the patient's involvement as an equal partner through their patient journey. ${ }^{53} \mathrm{~A}$ health technology can help improve health literacy and empower patients by 1 ) increasing patient and caregiver knowledge and understanding of the disease and treatment and/or 2) helping patients and caregivers to take an

Improved health literacy and empowerment active part in their treatment decisions and adherence.

Definition: This domain estimates the extent to which the technology gives the patient a better prediction/information on what happens to him/her (e.g. by reducing information asymmetry, improving understanding of treatment and target outcomes, empowering/enabling health literacy, health confidence, or prediction of likely responders to the treatment and reduction of individual variability) and therefore may reduce fear of the disease and/or risk of serious negative outcomes.

Incentive for manufacturers: To develop new technologies (including diagnostics or mobile applications) to inform, support and empower patients.

Justification for domain: Improvements in HRQOL are an important part of traditional value assessment, however, it is important to use patient-centered, disease-specific, validated PROs to ensure 1) PEx is captured beyond generic measurements of HRQOL, and 2) measurements are extended to the caregivers, in addition to patients. Caregivers are responsible for assisting patients through everyday life and have been categorized as a high-risk group due to unmet

Patient and caregiver reported outcomes needs and overlooked psychological burden. ${ }^{54}$ This domain also aims to capture the importance of community-centered care. $^{55}$

Definition: This domain represents the patient's and caregiver's general perception of how the illness and treatment affect the physical, physiological and social aspects of life. By using validated instruments related to PEx, outcomes are based on information coming directly from the patient or the caregiver without any interpretation by a clinician.

Incentive for manufacturers: 1 ) to consider and measure patient and caregiver meaningful outcomes related to PEx in the development of new technologies and 2) to generate data and measure these outcomes through validated instruments in real-world settings by asking patients and caregivers which outcomes are meaningful for them.

Justification for domain: A household's financial burden is an important consideration from the patient's perspective. Patients and caregivers may have to make trade-offs between financial commitments, the ability to work and paying for their portion of their treatment. Alternatively, a health technology may increase job productivity for either the patient or caregiver. When the evaluation of financial burden is conducted from a social perspective, direct and indirect costs of patients and caregivers should be considered. This domain aims to incentivize widespread collection and evaluation of these costs.

ousehold's

financial burden

Definition: This domain captures the financial burden of the disease/treatment for the patients and caregivers.

Incentive for manufacturers: 1 ) to consider the financial burden of disease/treatment for households when developing new technologies and 2) to measure this financial burden in real-world settings.

Justification for domain: An equity domain is often part of VFs, although the definition and measurement of equity is not frequently reported. This domain is intended to assess alternative therapies that may benefit vulnerable and/or neglected subpopulations of patients based on their socioeconomic status, type of disease or lack of research.

Improved access for vulnerable patient

Definition: This domain captures equity in timely access for vulnerable and neglected patient groups with unmet needs (e.g. rare disease patients, psychiatric patients/patients with mental health problems, pediatric patients, pregnant women, patients in rural areas). ${ }^{\text {a }}$

Incentive for manufacturers: 1) to provide solutions for the timely access to health technologies for vulnerable and neglected patient populations and 2) to measure the difference in the proportion of patients that achieve access to the therapy.

${ }^{a}$ Examples to be selected on a case-by-case basis.

$H R Q O L=$ health-related quality of life; $P E x=$ patient experience; $P R O s=$ patient reported outcomes; $V F=$ value framework. 


\section{TABLE 2 Indicative Measurement of PEx Domains}

\section{Criteria \\ Proposed measurement (to be finalized during national adaptation)}

- The assessed health technology shows no improvement in a) the level of considering patient's individual needs during treatment initiation or continuation and $b$ ) in the number of patients with improved choice.

Responsiveness to patients' individual needs

- The assessed health technology shows minor improvement in a) the level of considering patient's individual needs during treatment initiation or continuation and b) in the number of patients with improved choice.

- The assessed health technology shows major improvement in a) the level of considering patient's individual needs during treatment initiation or continuation and b) in the number of patients with improved choice.

Improved health literacy and empowerment

- The assessed health technology has no effect on health literacy and patient empowerment.

- The assessed health technology has a minor effect on improving health literacy and patient empowerment.

- The assessed health technology has a major effect on improving health literacy and patient empowerment.

- The assessed health technology shows no evidence on patient and caregiver reported outcomes and disease impact on patient's/caregiver's everyday life compared to current standard of care.

- The assessed health technology shows decrement on patient and caregiver reported outcomes and disease impact on patient's/caregiver's everyday life compared to current standard of care.

Patient and caregiver reported outcomes

- The assessed health technology shows no improvement on patient and caregiver reported outcomes and disease impact on patient's/caregiver's everyday life compared to current standard of care.

- The assessed health technology shows minor improvement compared to the minimal important difference/statistical difference but on average does not achieve a meaningful difference on patient and caregiver reported outcomes or disease impact on patient's/caregiver's everyday life compared to current standard of care.

- The assessed health technology shows major improvement compared to the minimal important difference/statistical difference and does, on average, achieve meaningful difference regarding patient and caregiver reported outcomes and disease impact on patient's/caregiver's everyday life compared to current standard of care.

- The assessed health technology has no evidence on the direct or indirect financial burden of the disease for the patient or the caregiver(s).

- The assessed health technology results in a major increase on the direct or indirect financial burden of the disease for the patient or the caregiver(s) which potentially compromises the sustainability of the household.

- The assessed health technology results in a minor increase on the direct or indirect financial burden of the disease for the patient or the caregiver(s) without a compromise on the sustainability of the household.

Household's financial burden

- The assessed health technology results in no increase on the direct or indirect financial burden of the disease for the patient or the caregiver(s).

- The assessed health technology results in a minor decrease on the direct or indirect financial burden of the disease for the patient or the caregiver(s).

- The assessed health technology results in a major decrease on the direct or indirect financial burden of the disease for the patient or the caregiver(s).

- There is no evidence whether the assessed health technology improves the timely access to the treatment of vulnerable or neglected patients.

Improved access for vulnerable patient populations

- The assessed health technology does not improve timely access to the treatment for vulnerable or neglected patients.

- The assessed health technology provides minor positive impact/improvement in timely access to the treatment of vulnerable or neglected patients.

- The assessed health technology provides major positive impact/improvement in timely access to the treatment of vulnerable or neglected patients.

$P E X=$ patient experience.

level of decision making could not be conducted. However, this study observed no signals that different types of health technologies (ie, pharmaceuticals, medical devices, combined technologies) should be assessed by a different set of PEx domains. Similarly, there was no evidence suggesting the need for different PEx domains for different levels of decision making. Conversely, the authors believe that PEx domains may be more impactful at the micro and mezzo levels of decision making once the decision to provide access has already been made at the macro level.

Practically, the next steps of implementation are likely to be through either creating a new, more holistic VF or updating an existing one with patient-relevant elements. ${ }^{40}$ While the definition of PEx domains has to be harmonized 
on a global scale, the relative weight and measurement of each PEx domain will always vary within and across countries, health technology types and decision-making levels according to patient opinion. The PEx field is moving very quickly and so the framework should be considered flexible and adaptable, depending on the requirements of specific HTA systems. Manufacturers of innovative technologies need to acknowledge that PEx may not be positive for all technologies; for some domains, a technology may not have any impact or could have a negative impact. To ensure feasibility and the support of decision makers, the policy implementation of the PEx domains is expected to be gradual, monitored using pilot phases and improved upon based on the accumulated experience and evidence, after which the final framework can be incorporated into the patient-centered value judgement of new technologies in different countries. ${ }^{40}$

Implementation will only be successful if there is consensus among key stakeholders, including healthcare professionals, patients and patient representatives with a selection of perspectives covering a range of disease types (eg, rare diseases) and demographics. These stakeholders could provide consensus through framework development committees. ${ }^{32}$ Furthermore, implementation would preferably be in partnership with influential supranational organizations or projects, to concentrate and maximize efforts. Successful implementation of the PEx framework needs to be evidence-based and data-driven. However, limited initial availability of PEx-related evidence should not block market access of new health technologies. Therefore, a potential future scenario could be to apply coverage with evidence development for technologies with claimed, but not solidly evidenced, PEx related benefits; a drug is given an initial license, but after the positive coverage decision the manufacturer must demonstrate in real world studies that the drug improves aspects of PEx to keep the level of reimbursement.

\section{LIMITATIONS}

This research has several limitations, some of which should be addressed by further research. Researchers only focused on published sets of domains, therefore information about payer decision making could not be retrieved from countries using a deliberative payer decision-making process. Additionally, the research and references included in this SLR and subsequent work are primarily focused on the non-emergency/elective setting. Further research is needed to evaluate the possibility of extending the framework to emergency medicine and to identify if any additional domains are necessary besides those proposed here.
A limited number of international payer experts challenged and validated the PEx framework; only two patient representatives with training in HTA were included in the development of the framework and at the expert panel discussion. To ensure the PEx framework is useful and relevant for both patients and payers, it could be pilot tested with larger panel discussions and (non-expert) focus groups of patients/patient representatives and payers during adaptation in different jurisdictions. However, the expert panel in this study included a range of international experts who were able to provide different perspectives on the framework.

Finally, further research is needed to explore any potential interrelation among PEx domains and between traditional value domains and PEx domains.

\section{Conclusions}

PEx is gaining increasing importance in HTA; however, current extensions of VFs related to PEx are heterogeneous and need standardization. Regulatory bodies can also drive standardization by defining PEx and its measurement. However, a framework to capture PEx may help provide consensus on PEx domains and the standards by which PEx can be measured in HTA. This SLR found little evidence to suggest there may be differences in the way PEx needs to be defined across different levels of decision making. Our subsequent work proposed criteria for such decisions going forward.

Investment in health technologies should explicitly consider patient centricity and societal value judgement to objectively assess patient and societal level value, and to increase both the transparency of decision making and the acceptance of these decisions. No improvement in the implementation of PEx domains can be expected without harmonizing with other initiatives with similar aims.

Healthcare systems are expected to gradually adapt the PEx domains, either formally or informally. The five proposed domains can be used to reduce the heterogeneity of PEx in extended VFs across jurisdictions by suggesting a standard set of PEx domains. Alternatively, the domains can be used to improve the patient centricity of those VFs in which PEx have not been considered, regardless whether PEx is taken into account in (1) deliberate decision-making processes, (2) augmented cost effectiveness analysis frameworks, or (3) MCDA tools. These domains could help manufacturers incorporate PEx during clinical development, and guide discussions between decision makers, providers and patients/patient experts/patient representatives throughout a decision-making process. 
Overall, this paper describes an attempt to harmonize PEx related value judgement in the evaluation of health technologies in different jurisdictions. It is our hope that multinational companies will consider standardized PEx domains in the evidence generation process of their new technologies.

\section{DISCLOSURES}

This study was funded and sponsored by UCB Pharma. The funding agreement ensured the authors' independence in designing the study, interpreting the data, writing, and publishing the report. Charokopou, Mountain, and Szegvari are employed by UCB Pharma. Inotai, Jakab, and Kalo are employed by Syreon Research Institute, which received funding from UCB Pharma for this research.

Brixner has received fees from AbbVie, Elevar, Millcreek Outcomes Group, Novartis, Sanofi, UCB Pharma, and Xcenda. Campbell has received grants and contracts from the PhRMA Foundation and the Institute for Clinical and Economic Review. During a sabbatical leave, Campbell collaborated with Syreon Research Institute on research projects that included funding from UCB Pharma. Hawkins has received consultancy fees from UCB Pharma. Kristensen has received speakers bureau fees from Pfizer, AbbVie, Amgen, UCB Pharma, Celgene, Bristol-Myers Squibb, MSD, Novartis, Eli Lilly, and Janssen Pharmaceuticals and consultancy fees from UCB Pharma.

\section{ACKNOWLEDGMENTS}

The authors thank the investigators and their teams who took part in this study. The authors acknowledge Frank Ulrich-Fricke, Technische Hochschule Nürnberg, Germany for input during the PEx framework development. The authors also acknowledge Susanne Wiegratz, UCB Pharma, Monheim am Rhein, Germany, and Simone E. Auteri, MSc EMS PhD, UCB Pharma, Brussels, Belgium, for publication coordination and Emma Phillips, PhD, and Simon Foulcer, PhD, Costello Medical, UK, for medical writing and editorial assistance based on the authors' input and direction and funded by UCB Pharma.

\section{REFERENCES}

1. Berger ML, Bingefors K, Hedblom EC, Pashos CL, Torrance GW, Smith MD. Health Care Cost, Quality, and Outcomes: ISPOR Book of Terms. ISPOR; 2003.

2. Velasco-Garrido M, Busse R. Health Technology Assessment: An introduction of objectives, role of evidence, and structure in Europe. European Observatory on Health Systems and Policies. Published 2005. Accessed October 02, 2019. http:// www.euro.who.int/ data/assets/pdf file/0018/90432/E87866.pdf

3. Food and Drug Administration. Guidance for Industry: Patient-Reported Outcomes Measures: Use in Medical Product Development to Support Label Claims. Published December 2009. Accessed January 12, 2021. https://www. fda.gov/media/77832/download

4. Whitehead SJ, Ali S. Health outcomes in economic evaluation: the QALY and utilities. British Medical Bulletin. 2010;96:5-21.

5. Food and Drug Administration. PatientFocused Drug Development: Collecting Comprehensive and Representative Input. Published 2018. Accessed January 14, 2021. https://www.fda.gov/regulatory-information/search-fda-guidance-documents/ patient-focused-drug-developmentcollecting-comprehensive-and-representative-input

6. Linthicum MT, dosReis S, Slejko JF, Mattingly TJ 2nd, Bright JL. The Importance of Collaboration in Pursuit of Patient-Centered Value Assessment. Patient. 2020. doi: 10.1007/ s40271-020-00446-3

7. Diaby V, Ali AA, Montero AJ. Value Assessment Frameworks in the United States: A Call for Patient Engagement. Pharmacoecon Open. 2019;3:1-3.

8. Marsh K, Ganz ML, Hsu J, Strandberg-Larsen M, Gonzalez RP, Lund N. Expanding Health Technology Assessments to Include Effects on the Environment. Value Health. 2016;19:249-54.
9. Angelis A, Kanavos P. Multiple Criteria Decision Analysis (MCDA) for evaluating new medicines in Health Technology Assessment and beyond: The Advance Value Framework. Soc Sci Med. 2017;188:137-56.

10. Garrison LP Jr., Neumann PJ, Willke RJ, et al. A Health Economics Approach to US Value Assessment Frameworks - Summary and Recommendations of the ISPOR Special Task Force Report [7]. Value Health. 2018;21(2):161-65.

11. Garrison LP, Jr., Pauly MV, Willke RJ, Neumann PJ. An Overview of Value, Perspective, and Decision Context - A Health Economics Approach: An ISPOR Special Task Force Report [2]. Value Health. 2018;21(2):124-30.

12. Schnipper LE, Davidson NE, Wollins DS, et al. Updating the American Society of Clinical Oncology Value Framework: Revisions and Reflections in Response to Comments Received. J Clin Oncol. 2016;34:2925-34.

13. Rocque G, Miller-Sonnet E, Balch A, et al. Engaging Multidisciplinary Stakeholders to Drive Shared DecisionMaking in Oncology. J Palliat Care. 2019;34(1):29-31.

14. Tamirisa NP, Goodwin JS, Kandalam A, et al. Patient and physician views of shared decision making in cancer. Health Expect. 2017;20:1248-53.

15. European Patients Forum. Strategic plan. Published 2019. Accessed October 02, 2019. http://www.eu-patient.eu/ library/Strategic-Plan/

16. European Commission. Horizon 2020 work programme from 2018 to 2020. Published 2018. Accessed October 02 , 2019. https://ec.europa.eu/programmes/ horizon2020/en/news/horizon2020-work-programme-2018-2020

17. Pharmaceutical Research and Manufacturers of America. Principles for value assessment frameworks. Published 2016. Accessed October 02, 2019. https:// www.phrma.org/codes-and-guidelines/ principles-for-value-assessment-frameworks 
18. National Institute for Health and Care Excellence. Patient experience in adult NHS services: improving the experience of care for people using adult NHS services. Published 2012. Accessed October 02, 2019. https://www.nice.org.uk/ guidance/cg138

19. Majid U, Visintini S. Patients' experiences with cardiac monitors for stroke, atrial fibrillation, and heart failure: a rapid qualitative review. CADTH rapid response reports. Canadian Agency for Drugs and Technologies in Health; 2018.

20. Food and Drug Administration. Plan for issuance of patient-focused drug development guidance. Published 2017. Accessed November 20, 2019. https:// www.fda.gov/media/105979/download

21. FasterCures. Patient-perspective value framework (PPVF). Published 2017. Accessed November 20, 2019. https:// www.fastercures.org/assets/Uploads/ PPVF-Version-1.0-Methodology-ReportFinal.pdf

22. Health Technology Assessment International. Changing HTA paradigms. Published 2016. Accessed October 02, 2019. https://htai.org/wp-content/ uploads/2018/02/PF_Background_ Paper 2016 FINAL.pdf

23. Institute for Clinical and Economic Review. Overview of the ICER value assessment framework and update for 2017-2019. Published 2017. Accessed May 21, 2021. https://34eyj51jerf417itp82ufdoewpengine.netdna-ssl.com/ wp-content/uploads/2020/10/ ICER-value-assessment-frameworkUpdated-050818.pdf

24. EUnetHTA. JA2 archive (2012-2015). Published 2018. Accessed October 02, 2019. https://www.eunethta.eu/ ja2-archive/

25. PREFER - Patient preferences. The patient perspective. Published 2019. Accessed January 7, 2020. https://www. imi-prefer.eu/

26. Center for Medical Technology Policy. Center for medical technology policy. Published 2019. Accessed October 08, 2019. http://www.cmtpnet.org/
27. Institute for Clinical and Economic Review. 2020-2023 Value assessment framework. Published 2020. Accessed January 21, 2021. http://icer. org/wp-content/uploads/2020/10/ ICER_2020_2023_VAF_102220.pdf

28. Marsh K, Caro JJ, Hamed A, Zaiser E. Amplifying each patient's voice: a systematic review of multi-criteria decision analyses involving patients. Appl Health Econ Health Policy. 2017;15:155-62.

29. Centers for Medicare and Medicaid. Oncology care model. Published 2019. Accessed October 10, 2019. https:// innovation.cms.gov/initiatives/ oncology-care/

30. Kolasa K, Zah V, Kowalczyk M. How can multi criteria decision analysis support value assessment of pharmaceuticals? - Findings from a systematic literature review. Expert Rev Pharmacoecon Outcomes Res. 2018;18:379-91.

31. Baltussen R, Niessen L. Priority setting of health interventions: the need for multi-criteria decision analysis. Cost Eff Resour Alloc. 2006;4:14.

32. Addario BJ, Fadich A, Fox J, et al. Patient value: Perspectives from the advocacy community. Health Expect. 2018;21(1):57-63.

33. Perfetto EM. ISPOR's initiative on US value assessment frameworks: a missed opportunity for ISPOR and patients. Value Health. 2018;21:169-70.

34. Wolf JA, Niederhauser V, Marshburn D, LaVela SL. Defining patient experience. Patient Exp J. 2014;1:3.

35. Liberati A, Altman DG, Tetzlaff J, et al. The PRISMA statement for reporting systematic reviews and meta-analyses of studies that evaluate healthcare interventions: explanation and elaboration. BMJ. 2009;339:b2700.

36. Frazao TDC, Camilo DGG, Cabral ELS, Souza RP. Multicriteria decision analysis (MCDA) in health care: a systematic review of the main characteristics and methodological steps. BMC Med Inform Decis Mak. 2018;18:90.
37. National Health Council. The Patient Voice in Value: The National Health Council Patient-Centered Value Model Rubric. Published 2016. Accessed April 02, 2020. https://nationalhealthcouncil. org/wp-content/uploads/2019/12/ValueRubric.pdf

38. Thokala P, Devlin N, Marsh K, et al. Multiple Criteria Decision Analysis for Health Care Decision Making - An Introduction: Report 1 of the ISPOR MCDA Emerging Good Practices Task Force. Value Health. 2016;19(1):1-13.

39. Marsh K, Jzerman IM, Thokala P, et al. Multiple Criteria Decision Analysis for Health Care Decision Making - Emerging Good Practices: Report 2 of the ISPOR MCDA Emerging Good Practices Task Force. Value Health. 2016;19:125-37.

40. Inotai A, Nguyen HT, Hidayat B, et al. Guidance toward the implementation of multicriteria decision analysis framework in developing countries. Expert Rev Pharmacoecon Outcomes Res. 2018;18(6):585-92.

41. Drake JI, de Hart JCT, Monleón C, Toro W, Valentim J. Utilization of multiple-criteria decision analysis (MCDA) to support healthcare decision-making FIFARMA, 2016. J Mark Access Health Policy. 2017;5(1):1360545.

42. de Greef-van der Sandt I, Newgreen D, Schaddelee M, et al. A quantitative benefit-risk assessment approach to improve decision making in drug development: Application of a multicriteria decision analysis model in the development of combination therapy for overactive bladder. Clin Pharmacol Ther. 2016;99(4):442-51.

43. Dankó D, Molnár MP. Balanced assessment systems revisited. J Mark Access Health Policy. 2017;5:1355190.

44. Kolasa K. Are pricing and reimbursement decision-making criteria aligned with public preferences regarding allocation principles in the Polish healthcare sector? Expert Rev Pharmacoecon Outcomes Res. 2014;14:751-62. 
45. van Amsterdam J, Nutt D, Phillips L, van den Brink W. European rating of drug harms. J Psychopharmacol. 2015;29:655-60.

46. Belgian Health Care Knowledge Centre. Incorporating societal preferences in reimbursement decisions. Published 2014. Accessed January 07, 2020. https://kce.fgov.be/sites/default/ files/atoms/files/KCE 234 reimbursement_decisions_Report_0.pdf

47. Williams P, Mauskopf J, Lebiecki J, Kilburg A. Using multicriteria decision analysis during drug development to predict reimbursement decisions. J Mark Access Health Policy. 2014;2:25270.

48. Janssens R, Huys I, van Overbeeke E, et al. Opportunities and challenges for the inclusion of patient preferences in the medical product life cycle: a systematic review. BMC Med Inform Decis Mak. 2019;19(1):189.
49. Tariman JD, Berry DL, Cochrane B, Doorenbos A, Schepp KG. Physician, patient, and contextual factors affecting treatment decisions in older adults with cancer and models of decision making: a literature review. Oncol Nurs Forum. 2012;39:E70-83.

50. Porter ME, Teisberg EO. Redefining health care: creating value-based competition on results. Harvard Business Review Press; 2006.

51. Montori VM, Brito JP, Murad MH. The optimal practice of evidence-based medicine: incorporating patient preferences in practice guidelines. JAMA. 2013;310:2503-04.

52. Ratzan S, Parker R, Selden C, Zorn M. National library of medicine current bibliographies in medicine: health literacy. National Institutes of Health; 2000.
53. Nielsen-Bohlman L, Panzer AM, Kindig DA. Health Literacy: A Prescription to End Confusion. National Academies Press; 2004

54. Hinds C. The needs of families who care for patients with cancer at home: are we meeting them? J Adv Nurs. 1985;10(6):575-81.

55. Miaskowski C, Zimmer EF, Barrett KM, Dibble SL, Wallhagen M. Differences in patients' and family caregivers' perceptions of the pain experience influence patient and caregiver outcomes. Pain. 1997;72:217-26. 